\title{
Inhaled Insulin-A New Insulin Delivery System
}

\section{Laura Zemany, MD and Martin J Abrahamson, MD$$
\text { a report by }
$$

\author{
Research Fellow and Medical Director \\ Joslin Diabetes Center, Harvard Medical School
}

DOI: 10.17925/USE.2006.00.1.48

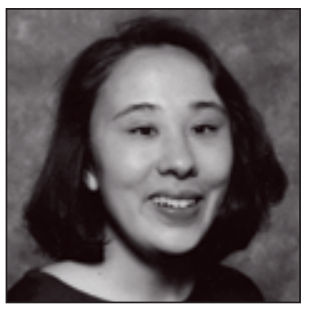

Laura Zemany, MD, is a Clinical Fellow in Endocrinology at the Beth Israel Deaconess Medical Center and the Joslin Diabetes Center. Dr Zemany received her medical degree at the Washington University Schoo of Medicine, and she completed he medicine at the Beth Israe Deaconess Medical Center in Boston, Massachusetts.

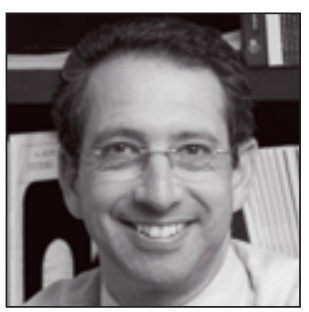

Martin J Abrahamson, MD, is Associate Professor of Medicine at Harvard Medical School and Medical Director and Senior Vice President of the Joslin Diabetes Center in Boston, Massachusetts. At Joslin Clinic, Dr Abrahamson is credited with reorganizing the Adult Diabetes Section into multidisciplinary teams comprised of physicians, nurse practitioners, nurse educators, nutritionists, and exercise physiologists. He co-directs the Joslin - Beth Israel Deaconess Medical Center (BIDMC) DiabetesHeart Program, a joint venture between Joslin Clinic and the Division of Cardiology at BIDMC. Dr Abrahamson also serves as Director

of the Division of Continuing Medical Education in the Department of Medicine. internship and residency in internal
The inhaled insulin, Exubera ${ }^{\circledR}$, was recently approved for the treatment of type 1 and type 2 diabetes. This article will review the delivery systems for inhaled insulin, the kinetics of inhaled insulin, clinical trials in type 1 and type 2 diabetes, and safety considerations for inhaled insulin.

\section{Delivery of Inhaled Insulin}

The lungs are an excellent target for the delivery of medications since the lungs have a highly permeable membrane, a large surface area $\left(140 \mathrm{~m}^{2}\right.$ in adults $)$, and a rich blood supply.1,2 Several factors affect where inhaled particles will be deposited in the respiratory system such as the size, solubility, and velocity of the particle. The new insulin delivery systems that have either been approved for clinical use or are in clinical trials use either a dry powder or a liquid formulation of insulin. ${ }^{3,4}$ The insulin can be administered to the lungs by an aqueous mist inhaler or a dry powder inhaler. Insulin delivery systems which are under investigation include Exubera (Pfizer, Nektar), AERx iDMS (Novo-Nordisk), Technosphere (Mannkind), and AIR (Eli Lilly, Alkermes). The device used for the delivery of Exubera uses a dry powder insulin, which contains insulin (60\%), mannitol, sodium citrate, and glycine. The insulin is packaged into $1 \mathrm{mg}$ or $3 \mathrm{mg}$ blisters, which contain approximately the equivalent of three or nine units of insulin. The blister is placed into the inhaler and, utilizing compressed air, the insulin is aerosolized into a transparent holding chamber. The patient views a cloud of aerosolized insulin, and during the beginning of a deep breath, the patient inhales the insulin through a mouthpiece.

\section{Pharmacokinetics}

Inhaled insulin has pharmacokinetic properties similar to the rapid acting insulin analogs and hence is used for premeal insulin coverage. The properties of inhaled insulin (Exubera 6mg), lispro insulin (18 units), and regular insulin (18 units) were determined in a euglycemic clamp study in 17 healthy male volunteers. ${ }^{5}$ The onset of action (time to reach 50\% maximal glucose infusion rate) was shorter for Exubera (32 minutes) compared to lispro and regular (41 minutes, $p<0.05$ and 48 minutes, $p<0.001$, respectively). The time to maximal effect was similar for inhaled insulin (143 minutes) and lispro (137 minutes), but it was shorter compared to regular (193 minutes, $\mathrm{p}<0.01$ ). The duration of action (time to reach late half maximal effect) was similar for inhaled insulin (387 minutes) and regular (415 minutes), but it was longer than lispro (313 minutes, $\mathrm{p}<0.01)$.

A similar study measured the dose response of lispro insulin and inhaled insulin delivered by the AIR particle technology. ${ }^{6}$ Different dosages of inhaled insulin $(2.6,3.6,5.2$, or $7.8 \mathrm{mg})$ and lispro insulin (6, 12 , or 18 units) were studied, and the study found that
1. Patton J S, "Unlocking the opportunity of tight glycaemic control. Innovative delivery of insulin via the lung", Diabetes Obes Metab (2005); 7 Suppl 1: pp. S5-8.

2. Barnett A H, "Exubera inhaled insulin: a review", Int J Clin Pract (2004);58(4): pp. 394-401.

3 Owens D R, "Alternative routes of insulin delivery", Diabetic Med (2003);20(11): pp. 886-898.

4. Cefalu WT, "Concept, strategies, and feasibility of noninvasive insulin delivery”, Diabetes Care (2004); 27(1): pp. $239-246$.

5. Rave K M, "Time-action profile of inhaled insulin in comparison with subcutaneously injected insulin lispro and regular human insulin”, Diabetes Care (2005); 28(5): pp. 1,077-1,082.

6. Rave $K M$, "Dose response of inhaled dry-powder insulin and dose equivalence to subcutaneous insulin lispro", Diabetes Care (2005); 28(10): pp. 2, 400-2, 405.

7. Brunner GA, "Dose-response relation of liquid aerosol inhaled insulin in type I diabetic patients", Diabetologia (2001); 44(3): pp. 305-308.

8. Kim D, "Dose-response relationships of inhaled insulin delivered via the Aerodose insulin inhaler and subcutaneously injected insulin in patients with type 2 diabetes", Diabetes Care (2003); 26(10): pp. 2,842-2,847.

9. Himmelmann A, "The impact of smoking on inhaled insulin", Diabetes Care (2003); 26(3): pp. 677-682. 
the relative bioavailability of inhaled insulin was consistent across different doses. Similar findings regarding the pharmacokinetics of inhaled insulin have been reported in subjects with type 1 and type 2 diabetes. ${ }^{7,8}$ The absorption of inhaled insulin is increased in smokers compared to nonsmokers, ${ }^{9,10}$ and patients with asthma absorb less insulin compared to healthy volunteers. ${ }^{11}$ to -0.01). In both studies, larger reductions in the fasting plasma glucose were observed in inhaled insulin group. For instance, in the second study, the mean adjusted change in the fasting plasma glucose was $-35 \mathrm{mg} / \mathrm{dl}$ in the inhaled insulin group compared to $+4 \mathrm{mg} / \mathrm{dl}$ in the subcutaneous group (treatment group difference $-39.5 \mathrm{mg} / \mathrm{dl}$, CI -57.5 to -21.6$)$. In both studies, the risk for hypoglycemia was slightly

\section{The lungs are an excellent target for the delivery of medications since the lungs have a highly permeable membrane, a large surface area $\left(140 m^{2}\right.$ in adults), and a rich blood supply.}

\section{Clinical Trials: Type I DM}

Several clinical studies in type 1 diabetes have evaluated the efficacy and tolerability of inhaled insulin. ${ }^{12}$ These studies have shown that inhaled insulin and subcutaneous insulin can achieve similar glycemic control. ${ }^{13}$ In a six-month study, 335 type 1 diabetics were randomized to prandial inhaled insulin and ultralente or regular and $\mathrm{NPH}$ insulin. ${ }^{14}$ The decrease in the $\mathrm{HbA}_{1 \mathrm{c}}$ was similar for inhaled insulin $(8.1 \%$ to $7.9 \%)$ and subcutaneous insulin $(8.1 \%$ to $7.7 \%$; adjusted treatment group difference $0.16 \%, 95 \%$ CI -0.01 to 0.32 ).

In a second six-month study, 328 type 1 diabetics were randomized to prandial inhaled insulin and twice daily $\mathrm{NPH}$ or regular insulin and twice daily NPH. ${ }^{15}$ The decrease in $\mathrm{HbA}_{1 \mathrm{c}}$ was similar for inhaled insulin $(8.0-7.7 \%)$ and subcutaneous insulin (7.9-7.8\%; adjusted treatment group difference $-0.16 \%$, CI -0.34 lower for inhaled insulin compared to subcutaneous insulin. However, in the second study, the risk for severe hypoglycemia was higher in the inhaled insulin group (6.5 versus 3.3 events per 100 patient-months; risk ratio 2.00 , CI 1.28 to 3.12 ).

An open-label crossover study compared inhaled insulin and subcutaneous insulin (lispro or regular) in 137 type 1 diabetics on glargine. ${ }^{16}$ Patients were given inhaled insulin and glargine for 12 weeks, followed by subcutaneous insulin and glargine for 12 weeks. The treatment order was reversed for some of the patients. There were no significant differences in the $\mathrm{HbA}_{1 \mathrm{c}}$ between the inhaled insulin and subcutaneous insulin groups, and the rate of hypoglycemia was similar between the two groups. However, the fasting blood glucose was lower in the inhaled insulin group, and the rate of nocturnal hypoglycemia was higher in the inhaled insulin group (4.2 versus 2.7 events per 30 days, $\mathrm{p}<0.001)$.

10. Becker $R H$, "The effect of smoking cessation and subsequent resumption on absorption of inhaled insulin", Diabetes Care (2006);29(2): pp. 277-282.

11. Henry $R R$, "Inhaled insulin using the AERx Insulin Diabetes Management System in healthy and asthmatic subjects", Diabetes Care (2003);26(3): pp. 764-769.

12. Scherbaum WA, "Unlocking the opportunity of tight glycaemic control. Inhaled insulin: clinical efficacy", Diabetes Obes Metab (2005); 7 Suppl 1: pp. S9-13.

13. Skyler J S, "Efficacy of inhaled human insulin in type 1 diabetes mellitus: a randomised proof-of-concept study", Lancet (2001);357(9253): pp. 331-335.

14. Quattrin T, "Efficacy and safety of inhaled insulin (Exubera) compared with subcutaneous insulin therapy in patients with type 1 diabetes: results of a 6-month, randomized, comparative trial", Diabetes Care (2004);27(11): pp. 2,622-2,627.

15. Skyler J S, "Use of inhaled insulin in a basal/bolus insulin regimen in type 1 diabetic subjects: a 6-month, randomized, comparative trial”, Diabetes Care (2005);28(7): pp. 1,630-1,635.

16. Garg $S$, "Efficacy and safety of preprandial human insulin inhalation powder versus injectable insulin in patients with type 1 diabetes”, Diabetologia (2006);49(5): pp. 891-899.

17. DeFronzo R A, "Efficacy of inhaled insulin in patients with type 2 diabetes not controlled with diet and exercise: a 12-week, randomized, comparative trial”, Diabetes Care (2005);28(8): pp. 1,922-1,928. 


\section{Clinical Trials-Type 2 DM}

Clinical trials with inhaled insulin have been conducted in subjects with type 2 diabetes previously treated with diet, oral agents, or insulin. In a three-month study, 145 type 2 diabetics suboptimally controlled on diet $\left(\mathrm{HbA}_{1 \mathrm{c}} 8-11 \%\right)$ were randomized to inhaled insulin or rosiglitazone, $4 \mathrm{mg}$ twice a day. ${ }^{17}$ The decrease in the $\mathrm{HbA}_{1 \mathrm{c}}$ was greater for inhaled insulin compared with rosiglitazone $(-2.3 \%$ versus $-1.4 \%$; adjusted treatment group difference $-0.89 \%, 95 \% \mathrm{CI}-1.23$ to -0.55$)$. Of note, $44 \%$ of patients in the inhaled insulin group achieved a $\mathrm{HbA}_{1 \mathrm{c}}$ less than $7.0 \%$ compared with $18 \%$ of the patients in the rosiglitazone group (odds ratio 4.43, 95\% CI 1.94 to 10.12$)$. significant improvement in the $\mathrm{HbA}_{1 \mathrm{c}}$ in the patients treated with inhaled insulin $(-1.9 \%$ for insulin plus two oral agents, $-1.4 \%$ for inhaled insulin alone, $-0.2 \%$ for two oral agents). $\mathrm{A} \mathrm{HbA}_{1 \mathrm{c}}$ of less then $7 \%$ was achieved in $32 \%$ of the patients on inhaled insulin and two oral agents (odds ratio 44.7, CI 6.0 to 335.2 ), 16.7\% of the patients on inhaled insulin alone (odds ratio 19.0, CI 2.5 to 145.8 ), and $1 \%$ of the patients on oral agents alone.

The use of inhaled insulin versus subcutaneous insulin has also been evaluated in type 2 diabetics. In a six-month trial, type 2 diabetics were randomized to inhaled insulin and ultralente or to subcutaneous regular and NPH insulin. ${ }^{20}$ The decrease in the hemoglobin $\mathrm{HbA}_{1 \mathrm{c}}$ was similar between the inhaled insulin and subcutaneous insulin

\section{In patients with type 2 diabetes, the availability of inhaled insulin allows for greater acceptance of insulin therapy.}

Inhaled insulin can improve glycemic control in type 2 diabetics suboptimally controlled on oral agents. In a sixmonth study, inhaled insulin and metformin were compared in 427 poorly controlled type 2 diabetics on a sulfonylurea. ${ }^{18}$ In the subset of patients with an initial $\mathrm{HbA}_{1 \mathrm{c}}$ greater than 9.5\%, the decrease in $\mathrm{HbA}_{1 \mathrm{c}}$ from baseline was greater for inhaled insulin compared with metformin $(-2.17 \%$ versus $-1.79 \%$; adjusted difference $-0.38 \%, 95 \%$ CI -0.63 to -0.14$)$. However, in the subset of patients with an initial $\mathrm{HbA}_{1 \mathrm{c}}$ from $8 \%$ to $9.5 \%$, the change in $\mathrm{HbA}_{1 \mathrm{c}}$ was similar for inhaled insulin and metformin. Another three-month study evaluated 309 type 2 diabetics $\left(\mathrm{HbA}_{1 \mathrm{c}} 8-11 \%\right)$ on two oral agents (a sensitizer and secretagogue). ${ }^{19}$ The patients were divided into three groups-inhaled insulin alone, inhaled insulin and two oral agents, or oral agents alone. There was a groups $(-0.7 \%$ versus $-0.6 \%$, adjusted difference $-0.07 \%$, 95\% CI -0.32-0.17). Hypoglycemic events were lower in the group treated with inhaled insulin (1.4 versus 1.6 events per patient month; risk ratio $0.89,95 \%$ CI $0.82-0.97$ ).

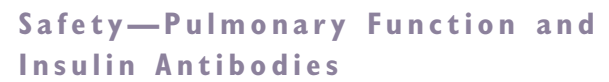

In all studies, patient satisfaction is greater in patients receiving inhaled insulin when compared to subcutaneous insulin. ${ }^{21-23}$ In patients with type 2 diabetes, the availability of inhaled insulin allows for greater acceptance of insulin therapy. ${ }^{24}$ However, there are two main safety considerations regarding the use of inhaled insulin-the effects on pulmonary function and the development of insulin antibodies. ${ }^{25}$

18. Barnett $A$, "An open, randomized, parallel-group study to compare the efficacy and safety profile of inhaled human insulin (exubera) with metformin as adjunctive therapy in patients with type 2 diabetes poorly controlled on a sulfonylurea" Diabetes Care (2006);29(6): pp. 1,282-1,287.

19. Rosenstock J, "Inhaled insulin improves glycemic control when substituted for or added to oral combination therapy in type 2 diabetes: a randomized, controlled trial”, Ann Intern Med (2005);143(8): pp. 549-558.

20. Hollander P A, "Efficacy and safety of inhaled insulin (Exubera) compared with subcutaneous insulin therapy in patients with type 2 diabetes: results of a 6-month, randomized, comparative trial", Diabetes Care (2004);27(10): pp. 2,356-2,362.

21. Gerber $R A$, "Treatment satisfaction with inhaled insulin in patients with type 1 diabetes: a randomized controlled trial”, Diabetes Care (2001);24(9): pp. 1,556-1,559.

22. Cappelleri J C, "Treatment satisfaction in type 2 diabetes: a comparison between an inhaled insulin regimen and a subcutaneous insulin regimen”, Clin Ther (2002);24(4): pp. 552-564.

23. Rosenstock J, "Patient satisfaction and glycemic control after 1 year with inhaled insulin (Exubera) in patients with type 1 or type 2 diabetes”, Diabetes Care (2004);27(6): pp. 1,318-1,323. 
Cough is more common with inhaled insulin therapy $(27 \%$ versus $5 \%) .{ }^{14}$ Some studies suggest a small but nonprogressive decline in pulmonary function in patients treated with inhaled insulin. ${ }^{14,20,27-28}$ In a sixmonth study in type 1 diabetics, a decrease in $\mathrm{DL}_{\mathrm{CO}}$ was noted in the inhaled insulin group. ${ }^{14}$ However, in a similar six-month study in type 2 diabetics, no significant differences in pulmonary function tests were noted in the inhaled insulin group. ${ }^{20}$ In a twoyear study in type 2 diabetics receiving inhaled insulin or a second oral agent, the change in $\mathrm{FEV}_{1}$ from baseline was greater in the inhaled insulin group at 24 weeks. ${ }^{27}$ However, this difference did not increase at 52 or 104 weeks. The differences in the $\mathrm{DL}_{\mathrm{CO}}$ were small between the two groups. Twelve weeks after
Several studies have shown that the prevalence of insulin antibodies is higher in patients receiving inhaled insulin. ${ }^{14}$ ${ }^{15,18-20}$ In a study of 47 patients with type 1 diabetes, the development of insulin antibodies did not affect the clinical safety or the pharmacodynamics of inhaled insulin..$^{29}$ An analysis of several phase II and III clinical trials revealed that patients given inhaled insulin developed a larger antibody response than patients given subcutaneous insulin, and the antibody response was largest for patients with type 1 diabetes..$^{30}$ Peak insulin antibody levels developed six to 12 months after exposure. The antibody response is non-neutralizing and predominantly of the $\mathrm{IgG}$ type. The presence of antibodies was not associated with changes in glycemic control, changes in insulin doses, hypoglycemic events,

\section{Given the efficacy and tolerability of inhaled insulin, inhaled insulin is a promising new delivery system that provides prandial insulin coverage, and it may ultimately play an important role in the future management of diabetes.}

discontinuing therapy, there were no significant differences in the $\mathrm{FEV}_{1}$ or $\mathrm{DL}_{\mathrm{CO}}$ between the two groups. In an extension study, pulmonary function studies were determined in 89 patients who completed four years of inhaled insulin therapy. ${ }^{28}$ In the patients receiving inhaled insulin, the annualized change in the $\mathrm{FEV}_{1}$ (mean +/- SE) was -0.057 +/$0.004 \mathrm{~L} / \mathrm{yr}$, and the annualized change in the DLCO was $-0.376+/-0.067 \mathrm{~mL} / \mathrm{min} / \mathrm{mmHg} / \mathrm{yr}$. In patients not receiving inhaled insulin (two-year data, $n=23$ ), the annualized change in the $\mathrm{FEV}_{1}$ was $-0.071+/$ $0.023 \mathrm{~L} / \mathrm{yr}$, and the annualized change in the $\mathrm{DL}_{\mathrm{CO}}$ was $-0.673+/-0.423 \mathrm{~mL} / \mathrm{min} / \mathrm{mmHg} / \mathrm{yr}$. Hence, pulmonary function tests should be obtained at baseline, at six months after starting insulin therapy, and annually thereafter. ${ }^{26}$ changes in pulmonary function tests, or the incidence of hypersensitivity reactions.

\section{Conclusions}

Several clinical trials in type 1 and type 2 diabetics have demonstrated the efficacy and safety of inhaled insulin, and shown that inhaled insulin and subcutaneous insulin can achieve similar glycemic control. Studies that have examined the side effects of inhaled insulin have shown that inhaled insulin is well tolerated, and its use is associated with increased patient satisfaction. Given the efficacy and tolerability of inhaled insulin, inhaled insulin is a promising new delivery system that provides prandial insulin coverage, and it may ultimately play an important role in the future management of diabetes.

24. Freemantle $N$, "Availability of inhaled insulin promotes greater perceived acceptance of insulin therapy in patients with type 2 diabetes", Diabetes Care (2005);28(2): pp. 427-428.

25. Brain J D, "Unlocking the opportunity of tight glycaemic control. Inhaled insulin: safety", Diabetes Obes Metab (2005);7 Suppl 1: pp. S14-S18.

26. Pfizer, Exubera Package Insert, 2006.

27. Dreyer $M$, "Efficacy and two-year pulmonary safety data of inhaled insulin as adjunctive therapy with metformin or glibenclamide in type 2 diabetes patients poorly controlled with oral monotherapy", Diabetologia (2004); 47 (Suppl. 1): p. A44.

28. Skyler J S, "Long-term sustained efficacy and safety of inhaled insulin after four years of continuous therapy", Diabetologia (2004); 47 (Suppl. 1): p. A311.

29. Fineberg $S E$, "Antibody response to inhaled insulin in patients with type 1 or type 2 diabetes. An analysis of initial phase II and III inhaled insulin (Exubera) trials and a two-year extension trial", J Clin Endocrinol Metab (2005);90(6): pp. 3,287-3,294.

30. Heise T, "The effect of insulin antibodies on the metabolic action of inhaled and subcutaneous insulin: a prospective randomized pharmacodynamic study”, Diabetes Care (2005);28(9): pp. 2,161-2,169. 